\title{
Graphite/epoxy composite for building Bipolar Plates
}

\author{
Luca Spinelli ${ }^{1, *}$, Fabrizio Roncaglia ${ }^{1, *}$, Roberto Biagi ${ }^{2,3}$, Alessandro di Bona ${ }^{3}$, Marcello Romagnoli ${ }^{4}$, and Adele Mucci $^{1}$ \\ ${ }^{1}$ Dipartimento di Scienze Chimiche e Geologiche, Università di Modena e Reggio Emilia, 41125 Modena, Italy \\ ${ }^{2}$ Dipartimento di Scienze Fisiche, Informatiche e Matematiche, Università di Modena e Reggio Emilia, 41125 Modena, Italy \\ ${ }^{3} \mathrm{CNR}$ - Istituto Nanoscienze - Centro S3, 41125 Modena, Italy \\ ${ }^{4}$ Dipartimento di Ingegneria "Enzo Ferrari", 41125 Modena, Italy
}

\begin{abstract}
Bipolar plates (BPs) are important components of Proton Exchange Membrane Fuel Cells (PEMFC). Graphite-epoxy composites, having a better corrosion resistance than metal-based BPs and better mechanical properties than graphite BPs, are a promising alternative. In this study, we tried to develop graphite-epoxy composites meeting the technical US DOE targets for 2020, with a proper choice of manufacturing conditions that ensure a good compromise between conductivity, flexural strength, and gas permeability. In particular, we studied the influence of the filler to binder ratio, changed the molding temperature and time, and investigated the effects of increasing pressure both on in-plane conductivity and on helium permeability. We found that both formulation and molding pressure are crucial in determining the permeability of the graphite-epoxy composites, whereas molding temperature and time seem to play a minor role.
\end{abstract}

\section{Introduction}

Hydrogen powered electric vehicles rely mainly on Proton Exchange Membrane Fuel Cells (PEMFC) that are reaching the commercialization phase. Bipolar plates (BPs) are important components of PEMFC where they distribute the gases (hydrogen and oxygen or air) uniformly, support the membrane electrode assemblies, collect and conduct electric current, connect the stacks, allow proper water management, remove heat and sustain the clamp pressure [1].

Graphite-based BPs can be used in place of the metalbased ones but suffer from inherent brittleness, while graphite-epoxy composites, combining a better corrosion resistance than metals and better mechanical properties than graphite, are emerging as promising alternative materials for BPs [1]. In our effort to develop graphiteepoxy composites meeting the US DOE technical targets for 2020 [2], we are searching for an optimal compromise between conductivity, flexural strength, and gas permeability. To this end, we are looking for synergistic combinations in the preparation phases (i.e., composite formulation, mixing, molding).

We already proved the evident advantages coming from wet mixing compared to the dry one [3]. In this work, the search spanned over different resin to filler ratios, and higher molding temperature and pressure. We found that the molding pressure and composition play a crucial role in determining the gas permeability of the formed BP, whereas in-plane conductivity (IPC) can be maintained at values well above the DOE targets.

\section{Materials and methods}

\subsection{Composite discs fabrication}

\subsubsection{Materials}

Diglycidylether of bisphenol A (DGEBA) resin D.E.R. $311^{\circledR} \quad(187 \mathrm{~g} / \mathrm{eq})$ was from Dow Chemicals; isophoronediamine (IPDA) was from Merck; GraphCOND $^{\circledR} 45 / 98$ X, D50 $75 \mu \mathrm{m}, 98 \%$ carbon $(G 45)$ was from LUH (www.luh.de); GraphTERM ${ }^{\circledR}$ 23/99.9, D50 18-25 $\mu \mathrm{m}, 99,9 \%$ carbon (G23) was from LUH; carbon black (CB) PBX 51, D50 $17 \mu \mathrm{m}$, was from Cabot.

\subsubsection{Mixing}

A $100 \mathrm{~mL}$ screw cap container was equipped with a mechanic stirrer using a three-blade propeller. Carbon black PBX51 (0.249 g), G23 (0.679 g) and G45 (3.322 g) and dichloromethane $(40 \mathrm{~mL})$ were introduced, and the solution was stirred at $360 \mathrm{rpm}$ for $15 \mathrm{~min}$. Then DGEBA epoxy resin $(0.612 \mathrm{~g}$, dissolved in $2 \mathrm{~mL}$ of dichloromethane) was added under stirring. Ten minutes later, IPDA $(0.138 \mathrm{~g}$, dissolved in $2 \mathrm{~mL}$ of dichloromethane) was slowly dropped while maintaining the mixing. The mixture was further stirred for $5 \mathrm{~min}$. The solvent was removed by means of a rotary vacuum evaporator and $1.00 \mathrm{~g}$ of the obtained powder was transferred in the mold and pressed as described below. This amount permits to prepare 4 replicates.

\footnotetext{
*Corresponding author: luca.spinelli@unimore.it;fabrizio.roncaglia@unimore.it
} 


\subsubsection{Molding}

Each dried powder sample $(1.00 \mathrm{~g})$ was pressed by means of a custom-made pressing machine (Graf S.p.A.) [3], The machine's mold $(2 \mathrm{~cm}$ id) can operate between room temperature and $160{ }^{\circ} \mathrm{C}$, and with an applied force up to $96 \mathrm{kN}$ (corresponding to a maximum of $306 \mathrm{MPa}$ ). Temperature, pressure, and time were managed and recorded by a custom-made software (Labview ${ }^{\mathbb{B}}$ based) via an interfaced PC. The obtained discs were post-cured in oven $\left(60^{\circ} \mathrm{C}\right)$ for 2 days.

\subsection{In-plane conductivity (IPC) measurements}

IPC was measured by means of a digital source meter (Keithley 2400) equipped with a four-point probe, as described [3]. Each sample was produced in four replicates and probed in eight different points, four per side. IPC $\left(\mathrm{S} \mathrm{cm}^{-1}\right)$ was obtained using equation (1) by correcting the measured resistivity $\rho(\Omega \mathrm{cm})$, accordingly to the geometry of the sample [4].

$I P C=\frac{1}{\rho} \quad$ where $\quad \rho=\frac{\pi}{\ln 2} \cdot F\left(\frac{t}{s}\right) \cdot \frac{V}{I} \cdot t$

where $t$ is the sample thickness $(\mathrm{cm}), \mathrm{s}$ is the distance between two probe contacts $(\mathrm{cm}), \mathrm{V}$ is the measured voltage $(\mathrm{mV})$, I is the measured current $(\mathrm{mA})$ and $\mathrm{F}(\mathrm{t} / \mathrm{s})$ is the correction factor tabulated by F.M. Smits [4].

\subsection{Permeation coefficient (HP) measurements}

Different studies use hydrogen to obtain the gas permeability of BP. Supported by its lower kinetic diameter [5], we opted to utilize helium instead of hydrogen at this stage, since it allows safer and simpler handling conditions.

The permeation coefficient (HP), defined as in ref. [6], was measured by means of a helium leak detector (BALZER HLT-150) equipped with a customized sample holder (schematic is shown in Figure 1). The detector works in high vacuum and is able to provide a quantitative measure of the helium flux. The sample is placed between two chambers: the first one connected to the detector and the second one attached to a line switched either to an external vacuum pump or to a helium bottle. Each sample was produced in four replicates and each one was tested.

The measurement procedure is described below: the sample is placed in the sample holder within two O-rings to guarantee the gas-tight closure. The area of the surface exposed to helium is kept always the same for all samples. The sample holder is then placed between the two chambers and the enclosure secured with an external mechanical lock. The detector was then switched to READ mode, it evacuates the second chamber and starts measuring the helium flux. At the same time, also the external vacuum pump is activated for evacuating the first chamber. When the detector reading stabilizes at the background value (about $2 * 10^{-9} \mathrm{sccm} \mathrm{s} \mathrm{s}^{-1}$ ) the first chamber was switched to the helium bottle and filled at 1 $\mathrm{atm}$. After some seconds needed to stabilize the reading, the helium flux is acquired.
HP was calculated according to equation (2), where the helium leak detector response is Ans, the flow of helium detected expressed in sccm s${ }^{-1}$; the exposed area of the sample (determined by the O-ring id) is A $\left(1.539 \mathrm{~cm}^{2}\right)$; the atmospheric pressure of helium in the corresponding chamber is $\mathrm{P}(101325 \mathrm{~Pa})$.

$$
H P\left[\operatorname{sccm}\left(s^{-1} \mathrm{~cm}^{-2} \mathrm{~Pa}^{-1}\right)\right]=\frac{A n s}{A \cdot P}
$$

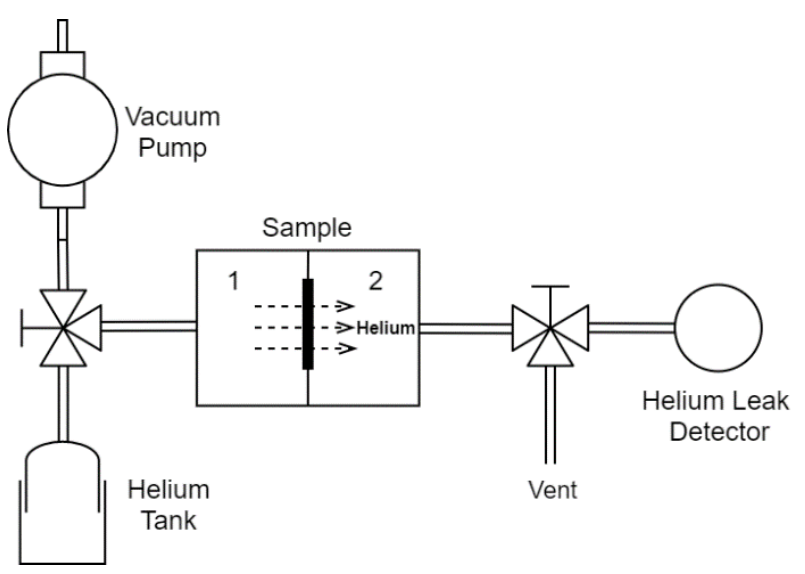

Fig. 1. Schematic of the helium leak detector and customized system to record permeation coefficient.

\subsection{Scanning electron microscopy (SEM)}

The surface morphology of the bipolar plates was investigated by a JEOL JSM-6010LA InTouchScope, using a back-scattered electron detector (BSED).

\section{Results and discussion}

In this work we mainly investigated the role played by composition and pressure used in the molding process on IPC and HP of graphite-epoxy composites for BPs. We started from a formulation with a filler to binder ratio of 91/9 (referred to as $\mathrm{C} 0$ in Table 1), molded at $80^{\circ} \mathrm{C}$ and $30 \mathrm{MPa}$ for $2 \mathrm{~h}$, very similar to that developed in a previous study [3] and that guarantees both good conductivity $\left(>160 \mathrm{~S} \mathrm{~cm}^{-1}\right)$ and flexural strength (nearly $50 \mathrm{MPa})$.

Table 1. Composition (percent by weight) of the composites used in the present study.

\begin{tabular}{|l|l|l|l|}
\hline & $\mathrm{C} 0(\% \mathrm{wt})$ & $\mathrm{C} 1(\% \mathrm{wt})$ & $\mathrm{C} 2(\% \mathrm{wt})$ \\
\hline G45 & 89.3 & 85 & 66.4 \\
\hline CB & 1.7 & 1.7 & 5.0 \\
\hline DGEBA & 7.3 & 12.4 & 12.2 \\
\hline IPDA & 1.7 & 3 & 2.8 \\
\hline G23 & - & - & 13.6 \\
\hline
\end{tabular}


Nevertheless, when we were able to measure the HP of discs of this composition, it resulted unacceptably high. Some tests on $\mathrm{C} 0$ samples, produced at increasing pressures, gave their best $\mathrm{HP}$ in the range $80-145 \mathrm{MPa}$, with a minimum value around $10^{-11} \mathrm{sccm}\left(\mathrm{s}^{-1} \mathrm{~cm}^{-2} \mathrm{~Pa}^{-1}\right)$ (the US DOE target for 2020 is $1.3 * 10^{-14} \mathrm{sccm}\left(\mathrm{s}^{-1} \mathrm{~cm}^{-2}\right.$ $\left.\mathrm{Pa}^{-1}\right)$ [2].

SEM micrographs, obtained with back-scattered electrons, showed that cracks were present around the graphite flakes (Figure 2). In addition, we did not observe an optimal reproducibility in conductivity performances.

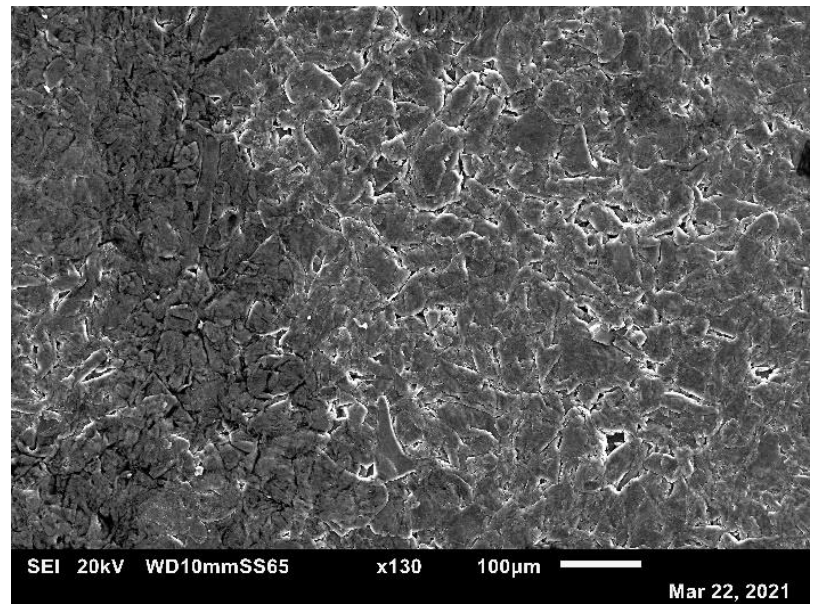

Fig. 2. SEM micrograph of a disc of composition $\mathrm{C} 0$ obtained at $26 \mathrm{MPa}$.

This scarce reproducibility was attributed mainly to the time elapsing between composite preparation and molding, that was then reduced shortening the molding time and enhancing the molding temperature. Shorter time and higher temperature and pressure are also closer to the usual industrial fabrication conditions for this class of materials and increase the production rate. To test both the effect of these different molding conditions and of a higher binder content, some tests were carried out on samples with a $15 \%$ of epoxy resin (composition $\mathrm{C} 1$, Table 1), at $150{ }^{\circ} \mathrm{C}$ and $160 \mathrm{MPa}$ for 5-10 min obtaining IPC of 160-180 S cm$~^{-1}$, very similar to that of discs of C0 or similar composition [3]. HP was found around $10^{-14}$ $\operatorname{sccm}\left(\mathrm{s}^{-1} \mathrm{~cm}^{-2} \mathrm{~Pa}^{-1}\right)$, improving of three orders of magnitude. At this stage, no evidence of detrimental effects on IPC and mechanical properties were observed by increasing molding temperature and pressure and decreasing molding time.

Then, we enhanced the carbon black content (to 5\%) to reduce the costs, introduced a secondary filler $(\mathrm{G} 23)$, thinner, cheaper than the primary one (G45) and optimized for thermal conductivity, in the view of future studies on thermal conductivity and of industrial applications. After further tests, we decided to work at $t=$ $5 \mathrm{~min}, T=150{ }^{\circ} \mathrm{C}, P<200 \mathrm{MPa}$ and $13.6 \%$ of $\mathrm{G} 23$ (composition C2, Table 1). This change in formulation did not improve IPC or HP but improved the preparation, because it allowed the reduction of about $30 \%$ of the amount of solvent required to obtain a proper dispersion while mixing. We focussed then on the effects of increasing pressure on IPC and HP on samples with this last composition (C2). We observed that with the new setup IPC is almost independent from pressure (Figure 3), except at the lowest values $(<60 \mathrm{MPa})$. A narrow IPC range is observed at $\mathrm{P}>60 \mathrm{MPa}\left(170 \pm 4 \mathrm{~S} \mathrm{~cm}^{-1}\right)$, meaning that the integrity of the conductive network is well maintained in a broad range of pressure. Interestingly, IPC shows a linear correlation with density $\left(R^{2}=0.97\right.$, Figure 4) and, as expected, a higher density results in a more efficient conducting network.

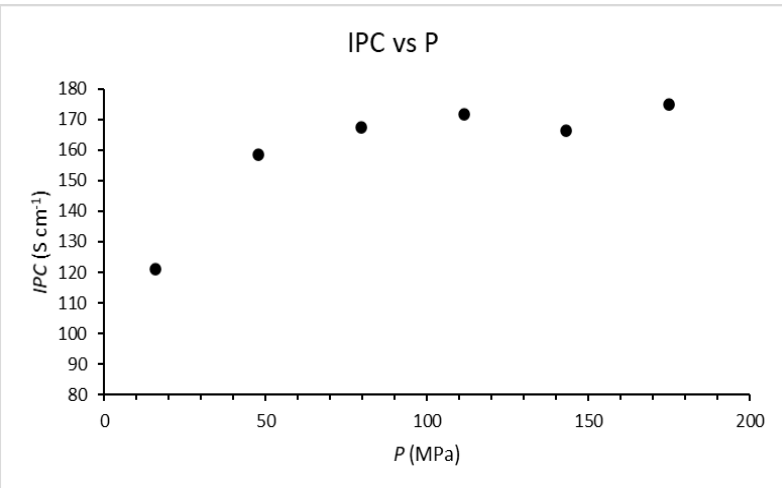

Fig. 3. IPC of discs of composition $\mathrm{C} 2$ obtained at increasing molding pressures.

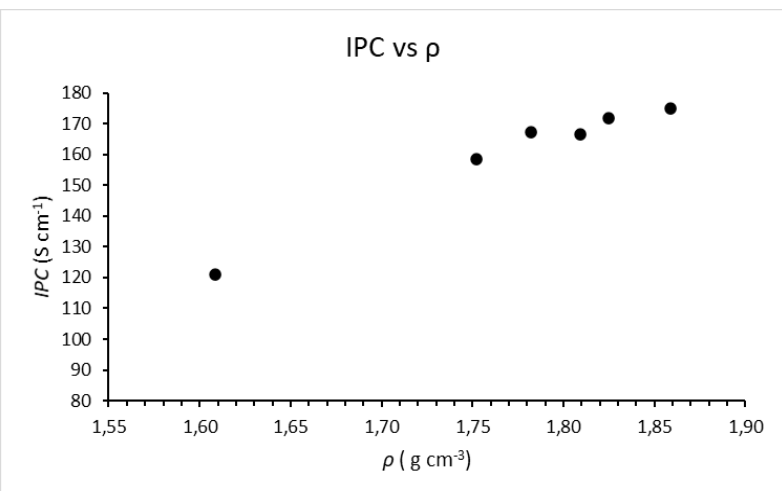

Fig. 4. IPC of discs of composition C2 vs density $(\rho)$.

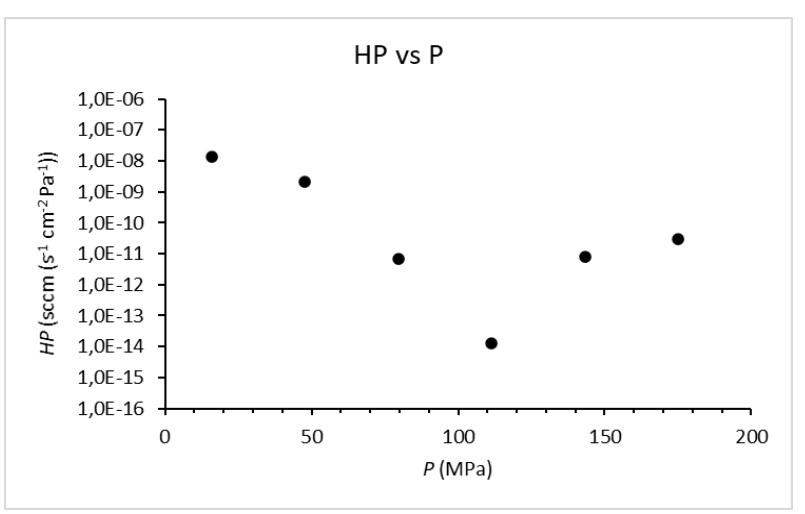

Fig. 5. Helium permeability (HP, logarithmic scale) of discs of composition $\mathrm{C} 2$ obtained at increasing molding pressures.

At a $15 \%$ of binder and with $5 \%$ of carbon black and $13.6 \%$ of G23 (C2) the HP of the discs had still a huge decrease of 1-3 orders of magnitude (Figure 5) with respect to that of samples of composition $\mathrm{C} 0$, reaching values close to the permeability targets [2]. These results 
show that good permeability is obtainable at intermediate pressure. The best result was obtained at $111 \mathrm{MPa}$ where the permeability reaches values around $1.2 * 10^{-14} \mathrm{sccm}\left(\mathrm{s}^{-}\right.$ $\left.{ }^{1} \mathrm{~cm}^{-2} \mathrm{~Pa}^{-1}\right)$. Nevertheless, the fluctuations of HP observed for similar pressure values, indicates that there is still room for improvement of the standardization of the whole fabrication/testing process.

\section{Conclusions}

High electric conductivity and low gas permeability are among the most important characteristics required to BPs to be used in PEMFC, together with good mechanical performances. In this study, we showed that a moderate increase in the epoxy resin content, the use of mixtures of properly chosen fillers and of appropriate pressure hugely improves the permeability of the composite, several orders of magnitude, while maintaining IPC values well above US DOE targets $\left(>100 \mathrm{~S} \mathrm{~cm}^{-1}\right)$. This is probably due to the fact that amounts of resin too small are not able to wet properly the graphite flakes, leaving void paths that permit gas diffusion, whereas addition of a filler with a low granulometry helps in better distributing the binder. Molding pressures in the range 110-160 MPa and epoxy resin around $15 \%$ seem to be appropriate to obtain low gas permeability. Carbon black amounts up to $5 \%$ are compatible with low HP and good IPC values. In addition, short cycle production conditions (high temperatures and short times) seem not to influence significantly neither IPC nor HP. Further optimization of the formulation and fabrication process are in progress to improve the reproducibility of the properties of the graphite-epoxy resin composites.

\section{Acknowledgments}

The authors are thankful to Fondazione Cassa di Risparmio di Modena for funding the CARCOM project (grant LINEAFCRM2019FARINTERDMUCCI).

\section{References}

1. N. Saadat, H.N. Dhakal, J. Tjong, S. Jaffer, W. Yang, M. Sain, Renew. Sustain. Energy Rev. 138, 110535, (2021)

2. Hydrogen and Fuel Cell Technologies Office, Electrolyte Membrane Fuel Cell Components, https://www.energy.gov/eere/fuelcells/doetechnical-targets-polymer-electrolyte-membranefuel-cell-components

3. F. Roncaglia, M. Romagnoli, S. Incudini, E. Santini, M. Imperato, L. Spinelli, A. di Bona, R. Biagi, A. Mucci, Int. J. Hydrogen Energy, 46, 4407, (2021)

4. F.M. Smits. Bell Syst. Tech. J. 37, 711 (1958)

5. S. Matteucci, Y. Yampolskii, B.D. Freeman, I. Piannau, Transport of Gases and Vapors in Glassy and Rubbery Polymers chapter 1 (John Wiley \& Sond, Ltd, Eds: Y. Yampolskii, B.D. Freeman, I. Piannau, 2006)

6. R. Blunk, F. Zhong, J. Owens, Journal of Power Sources, 159, 533 (2006) 